\title{
Biomarkers Predictive of Exacerbations in the SPIROMICS and COPDGene Cohorts
}

Jason D. Keene ${ }^{1}$, Sean Jacobson ${ }^{2}$, Katerina Kechris ${ }^{3}$, Gregory L. Kinney ${ }^{3}$, Marilyn G. Foreman ${ }^{4}$, Claire M. Doerschuk ${ }^{5}$, Barry J. Make ${ }^{2}$, Jeffrey L. Curtis ${ }^{6,7}$, Stephen I. Rennard ${ }^{8 *}$, R. Graham Barr ${ }^{9}$, Eugene R. Bleecker ${ }^{10}$, Richard E. Kanner ${ }^{11}$, Eric C. Kleerup ${ }^{12}$, Nadia N. Hansel ${ }^{13}$, Prescott G. Woodruff ${ }^{14}$, MeiLan K. Han ${ }^{6}$, Robert Paine ${ } I^{11}{ }^{11}$, Fernando J. Martinez ${ }^{15}$, Russell P. Bowler ${ }^{2}$, and Wanda K. O’Neal ${ }^{5}$; for the COPDGene and SPIROMICS Investigators ${ }^{\ddagger}$

${ }^{1}$ University of Colorado Anschutz Medical Campus, Aurora, Colorado; ${ }^{2}$ National Jewish Health, Denver, Colorado; ${ }^{3}$ Department of Biostatics and Informatics, Colorado School of Public Health, University of Colorado Anschutz Medical Campus, Aurora,

Colorado; ${ }^{4}$ Department of Medicine, Morehouse School of Medicine, Atlanta, Georgia; ${ }^{5}$ Marsico Lung Institute/Cystic Fibrosis Research Center, Department of Medicine, University of North Carolina at Chapel Hill, Chapel Hill, North Carolina; ${ }^{6}$ Division of Pulmonary and Critical Care Medicine, University of Michigan Health System, Ann Arbor, Michigan; ${ }^{7}$ VA Ann Arbor Healthcare System, Ann Arbor, Michigan; ${ }^{8}$ Division of Pulmonary and Critical Care Medicine, University of Nebraska, Omaha, Nebraska; ${ }^{9}$ Department of Medicine, Columbia University Medical Center, New York, New York; ${ }^{10}$ Center for Genomics and Personalized Medicine Research, Wake Forest School of Medicine, Winston-Salem, North Carolina; ${ }^{11}$ Department of Internal Medicine, Division of Pulmonary and Critical Care Medicine, University of Utah, Salt Lake City, Utah; ${ }^{12}$ Division of Pulmonary and Critical Care Medicine, Department of Medicine, David Geffen School of Medicine, University of California Los Angeles, Los Angeles, California; ${ }^{13}$ Division of Pulmonary and Critical Care Medicine, Johns Hopkins University School of Medicine, Baltimore, Maryland; ${ }^{14}$ Division of Pulmonary, Critical Care, Sleep and Allergy, Department of Medicine and Cardiovascular Research Institute, University of California San Francisco, School of Medicine, San Francisco, California; and ${ }^{15}$ Department of Medicine, Weill Cornell Medical College, New York-Presbyterian Hospital/Weill Cornell Medical Center, New York, New York

\begin{abstract}
Rationale: Chronic obstructive pulmonary disease exacerbations are associated with disease progression, higher healthcare cost, and increased mortality. Published predictors of future exacerbations include previous exacerbation, airflow obstruction, poor overall health, home oxygen use, and gastroesophageal reflux.
\end{abstract}

Objectives: To determine the value of adding blood biomarkers to clinical variables to predict exacerbations.

Methods: Subjects from the SPIROMICS (Subpopulations and Intermediate Outcomes Measures in COPD Study) $(\mathrm{n}=1,544)$ and COPDGene (Genetic Epidemiology of COPD) $(n=602)$ cohorts had 90 plasma or serum candidate proteins measured on study entry using Myriad-RBM multiplex panels. We defined total exacerbations as subject-reported worsening in respiratory health requiring therapy with corticosteroids and/or antibiotics, and severe exacerbations as those leading to hospitalizations or emergency room visits. We assessed retrospective exacerbations during the 12 months before enrollment and then documented prospective exacerbations in each cohort. Exacerbations were modeled for biomarker associations with negative binomial regression including clinical covariates (age, sex, percent predicted $\mathrm{FEV}_{1}$, self-reported gastroesophageal reflux, St. George's Respiratory Questionnaire score, smoking status). We used the Stouffer-Liptak test to combine $P$ values for metaanalysis.

Measurements and Main Results: Between the two cohorts, 3,471 total exacerbations (1,044 severe) were reported. We identified biomarkers within each cohort that were significantly associated with a history of exacerbation and with a future exacerbation, but there was minimal replication between the cohorts. Although established clinical features were predictive of exacerbations, of the blood biomarkers only decorin and $\alpha_{2}$-macroglobulin increased predictive value for future severe exacerbations.

Conclusions: Blood biomarkers were significantly associated with the occurrence of exacerbations but were not robust between cohorts and added little to the predictive value of clinical covariates for exacerbations.

Keywords: COPD; exacerbation; biomarker

(Received in original form July 1, 2016; accepted in final form August 30, 2016)

${ }^{*}$ Current address: Early Clinical Development, Innovative Medicines, AstraZeneca, Melbourn, Hertfordshire, UK.

${ }^{\ddagger} A$ complete list of members may be found before the beginning of the REFERENCES.

Correspondence and requests for reprints should be addressed to Russell P. Bowler, M.D., Ph.D., Department of Medicine, Division of Pulmonary Medicine, National Jewish Health, Denver, CO 80206. E-mail: bowlerr@njhealth.org

This article has an online supplement, which is accessible from this issue's table of contents at www.atsjournals.org

Am J Respir Crit Care Med Vol 195, Iss 4, pp 473-481, Feb 15, 2017

Copyright $\odot 2017$ by the American Thoracic Society

Originally Published in Press as DOI: 10.1164/rccm.201607-13300C on August 31, 2016

Internet address: www.atsjournals.org 


\section{At a Glance Summary}

\section{Scientific Knowledge on the \\ Subject: Chronic obstructive} pulmonary disease (COPD) is a systemic disease, and recent studies suggest candidate blood biomarkers (e.g., C-reactive protein, fibrinogen, blood eosinophils, and white blood cell count) may be predictive of future exacerbations. However, few studies have reported large biomarker panels in multiple well-characterized COPD populations that also include smokers at risk for COPD and longitudinal data.

\section{What This Study Adds to the}

Field: This study examined blood biomarkers predictive of acute respiratory exacerbations in current and former smokers with and without COPD using two large, wellcharacterized cohorts, SPIROMICS (Subpopulations and Intermediate Outcomes Measures in COPD Study) and COPDGene (Genetic Epidemiology of COPD). Although within each cohort a subset of biomarkers was associated with the presence of exacerbations independent of clinical predictors (respiratory symptoms, airflow obstruction, gastrointestinal reflux, smoking status, history of prior exacerbation), replication of biomarkers across the two cohorts was poor, and the increased predictive value of adding biomarkers to clinical variables was small. The study highlights the need for cohort replication and improved understanding of COPD exacerbations.

Chronic obstructive pulmonary disease (COPD) is a spectrum of lung diseases characterized by airflow obstruction, emphysema, and chronic bronchitis. Some individuals with COPD experience acute exacerbations of COPD (AECOPD) typified by increased cough, sputum production, shortness of breath, and wheezing and are treated with antibiotics and/or prednisone. Recent work suggests that there are subsets of current and former smokers who are more susceptible to frequent AECOPD $(1,2)$. Clinical predictors of AECOPD include exacerbation during the preceding year, spirometric airflow obstruction, low respiratory health scores, and reported gastroesophageal reflux $(1,3,4)$.

However, the sensitivity and specificity of these clinical predictors is imperfect. Identifying blood-based biomarkers that could improve the prediction of AECOPD is an ongoing research focus that could potentially deliver precision medicine to this heterogeneous disease and increase the efficiency of clinical trials. Susceptible patients seem to exhibit greater systemic inflammation, as evidenced by elevated white blood cell count (1), fibrinogen $(5,6)$, IL-6 (7), and C-reactive protein (CRP) (6). Surfactant protein $\mathrm{D}$, fetuin $\mathrm{A}$, adiponectin, and CRP are implicated as predictive of AECOPDs (8-10). The U.S. Food and Drug Administration recently qualified plasma fibrinogen as a biomarker, primarily based on data from ECLIPSE (Evaluation of COPD Longitudinally to Identify Predictive Surrogate Endpoints), to enrich clinical trials for patients likely to have hospitalized COPD exacerbations (11). Despite these advances, blood biomarkers have not achieved widespread use or proven clinical benefit to predict AECOPD.

SPIROMICS (Subpopulations and Intermediate Outcomes Measures in COPD Study) and COPDGene (Genetic Epidemiology of COPD) are two large, National Institutes of Health (NIH)-funded COPD cohorts in which retrospective exacerbations and peripheral blood for biomarker studies were collected at study entry and prospective exacerbations are being captured. To confirm published findings and identify novel biomarker associations with AECOPD, we generated a custom biomarker panel and evaluated the ability of measured biomarkers to predict AECOPD. Considering the cohorts individually and in a metaanalysis, our results call into question the reproducibility and clinical utility of blood biomarkers measured in the stable state to predict future COPD exacerbations.

\section{Methods}

\section{Study Population}

SPIROMICS (ClinicalTrials.gov Identifier: NCT 01969344) is an ongoing multicenter prospective observational study funded by the NIH (12) that enrolled 2,982 subjects between November 2011 and January 2015 The institutional review board at all participating sites approved the study protocol. Study participants provided written informed consent. The data reported here include results from the first 1,544 subjects enrolled in strata 2-4 (see APPENDIX in the online supplement for further details) $(12,13)$.

The NIH sponsored multicenter COPDGene (ClinicalTrials.gov Identifier: NCT 01969344) study was approved and reviewed by the institutional review board at all participating centers (14). Study participants provided written informed consent. In brief, all subjects were between 45 and 80 years old, had at least 10 packyears of smoking, and had not had an acute respiratory exacerbation for at least 30 days $(14,15)$. Of the larger cohort of 10,300 subjects, 602 were selected to undergo a comprehensive biomarker study. For more details on study population, please see online supplement APPENDIX.

\section{Biomarker Panel and Data Generation}

Based on the literature, we created a custom panel assay for 119 candidate biomarkers

Supported by NHLBI (R01HL 095432, R01 HL089856, and R01 HL089897) and National Center for Research Resources/National Institutes of Health (UL1 RR025680). SPIROMICS (Subpopulations and Intermediate Outcomes in COPD Study) is funded by contracts from the NHLBI (HHSN268200900013C, HHSN268200900014C, HHSN268200900015C, HHSN268200900016C, HHSN268200900017C, HHSN268200900018C, HHSN2682009000019C, and HHSN268200900020C), supplemented by contributions made through the Foundation for the National Institutes for Health from AstraZeneca; Bellerophon Therapeutics; Boehringer-Ingelheim Pharmaceuticals, Inc.; Chiesi Farmaceutici SpA; Forest Research Institute, Inc.; GSK; Grifols Therapeutics, Inc.; Ikaria, Inc.; Nycomed GmbH; Takeda Pharmaceutical Company; Novartis Pharmaceuticals Corporation; Regeneron Pharmaceuticals, Inc.; and Sanofi.

Author Contributions: J.D.K. had full access to all of the data in the study and takes responsibility for the integrity of the data and accuracy of the analysis. J.D.K., F.J.M., M.K.H., R.P.B., and W.K.O. contributed to the conception and design of the study. R.P.B., B.J.M., J.L.C., S.I.R., R.G.B., E.R.B., E.C.K., P.G.W., M.G.F., C.M.D., N.N.H., M.K.H., R.E.K., R.P., and F.J.M. contributed to the acquisition of the data. J.D.K., S.J., K.K., R.P.B., and W.K.O. contributed to the drafting of the manuscript. J.D.K., K.K., R.P.B., W.K.O., N.N.H., M.K.H., G.L.K., and J.L.C. contributed to revisions of the manuscript for critically important intellectual content. All of the authors approved this version of the manuscript to be published. 
using 13 Myriad-RBM (Austin, TX) multiplex assays (see Table E1 in the online supplement). Biomarkers were selected based on known or putative links to COPD; details of assay characteristics regarding coefficient of variation, detection range, and reliability estimates are described in a SPIROMICS pilot study (16) and a COPDGene study of emphysema (17), with additional details given by Sun and colleagues (18). All measurements used in this study were performed on a single blood sample collected at baseline. SPIROMICS also included a smaller study embedded in the larger study that used replicate samples collected at a separate visit to evaluate assay performance (Table E1).

Of the 119 measured biomarkers, 90 had values from at least $90 \%$ of subjects above the lower limit of quantitation (LLOQ) in both cohorts, and these form the basis of this analysis. One biomarker (prostate-specific antigen, free) was removed because it was only expressed in men.

\section{Clinical Data and Definitions}

Full details about COPDGene and SPIROMICS and the collection of clinical data has been described $(12,14)$. In both cohorts, COPD was defined as postbronchodilator ratio of $\mathrm{FEV}_{1}$ to $\mathrm{FVC}$ less than 0.70. We used Global Initiative for Chronic Obstructive Lung Disease (GOLD) guidelines (19) to assess COPD severity as GOLD stage 1-4. Current or ex-smokers at risk for COPD but without spirometric evidence of airflow obstruction $\left(\mathrm{FEV}_{1} / \mathrm{FVC}\right.$ $\geqslant 0.70$ ) were classified as GOLD stage 0 . Subjects with $\mathrm{FEV}_{1} / \mathrm{FVC}$ greater than or equal to 0.70 and $\mathrm{FEV}_{1}$ less than $80 \%$ were classified as preserved ratio impaired spirometry (20). Both studies used the St. George's Respiratory Questionnaire (SGRQ) to capture respiratory symptoms (21). Chronic bronchitis was defined in the COPDGene cohort as affirming either having chronic cough or phlegm production more than 3 months/yr for 2 or more consecutive years (22). Chronic bronchitis was defined in the SPIROMICS study by similar criteria. Volumetric computed tomography scans were acquired in both cohorts (see APPENDIX in the online supplement for further details related to clinical phenotyping) (14).

Acute exacerbations of respiratory disease were ascertained for the year prior at time of study entry; these were defined as retrospective exacerbations, and blood was drawn at this time. Prospective exacerbations were then monitored during the longitudinal follow-up visits; these encounters were scheduled every 6 months in COPDGene and every 3 months in SPIROMICS. An exacerbation was recorded when a patient reported a worsening of their respiratory status and were treated with antibiotics and/or systemic steroids for the episode irrespective of their treatment location. The total number of exacerbations was the total number of these events reported. A severe exacerbation was a report of visiting an emergency room and/or hospitalization for an acute episode of respiratory disease.

\section{Statistical Analysis}

Biomarker values were transformed individually for each study using empirical quantile normalization as described (18).

No analysis was conducted on analytes with greater than $90 \%$ of measurements less than LLOQ (Table E1). Linear regression models were conducted on analytes in which less than $10 \%$ of measurements were less than LLOQ. Logistic regression was conducted on for analytes with $10-90 \%$ of values less than LLOQ. Values below the LLOQ were imputed to half the LLOQ. The very small number $(<0.1 \%)$ of measurements above the detection range of the assay was set at the highest detected value for analysis.

Analyses were conducted using R (23). Exacerbations in the year before enrollment (retrospective) and during longitudinal followup (prospective) were modeled with a negative binomial regression with offset for exposure time and a zero inflation model to account for the excess number of subjects who reported no acute episodes of respiratory disease.

Associations of exacerbations to biomarkers were first determined without covariates. Then, this model was applied separately to the subjects in the two study populations described previously for both COPDGene and SPIROMICS with the clinical covariates of age, sex, percent-predicted $\mathrm{FEV}_{1}$, self-report of gastroesophageal reflux, SGRQ, smoking status, and biomarker batch analysis (for SPIROMICS only). The zero-inflation component of the model included the clinical covariate percent-predicted $\mathrm{FEV}_{1}$ only. Clinical covariates were selected after reviewing the current literature on exacerbations; only clinical covariates that were significant in several cohort studies were used.
For each biomarker, the model fitting returns an estimate, $\mathrm{SE}$, and $P$ value for the association of the biomarker within the count model (negative binomial) and an estimate for the association of the biomarker for zero counts within the zeroinflated component of the model (gamma parameter) $(24,25)$. The $P$ values were adjusted for multiple testing using the Benjamini-Hochberg method and false discovery rate (FDR) values less than 0.05 were considered significant. The StoufferLiptak test was used to combine the $P$ values from each cohort for the metaanalysis. Prospective exacerbations were modeled using the same negative binomial regression offset for time and a zero inflation model; the same clinical covariates were used as in the retrospective analysis with the addition of adjusting for history of prior exacerbation.

Cox proportional hazards multiple regression were used to determine hazard ratios for time to first episode on significant biomarkers identified in the prospective analysis $(26,27)$. For the proportional hazards model, the clinical covariates from the negative binomial regression were used with the addition of history of exacerbation.

To estimate the effect size for the clinical predictors and biomarkers the Cragg and Uhler pseudo R-squared method was used on the zero-inflated negative binomial model (28).

A stepwise multinomial regression using significant univariate clinical factors and biomarkers was performed on both cohorts to assess for reproducibility between the cohorts (see ApPENDIX in the online supplement).

\section{Results}

\section{Study Population}

Demographics, physiology, healthscores, and quantitative computed tomography measurements for emphysema are listed in Table 1 for both cohorts. The cohorts were similar in many aspects, including age at enrollment, body mass index, pack-years of smoking history, and chronic bronchitis history (Table 1), with notable differences. SPIROMICS subjects were more likely to report being current smokers, were less likely to be non-Hispanic white, exhibited less airflow obstruction, but a greater bronchodilator response, higher SGRQ total score, lower BODE index, and higher resting oxygen saturation (all comparisons, 
Table 1. Characteristics of Cohorts

\begin{tabular}{|c|c|c|c|}
\hline & $\begin{array}{c}\text { COPDGene } \\
(n=602)\end{array}$ & $\begin{array}{c}\text { SPIROMICS } \\
(n=1,544)\end{array}$ & $P$ Value \\
\hline Age at enrollment, yr & $63.6(8.6)$ & $64.5(8.8)$ & 0.03 \\
\hline $\mathrm{BMI}$ & $28.3(5.5)$ & $27.9(5.3)$ & 0.13 \\
\hline Male, \% & 51 & 55 & 0.12 \\
\hline Current smoker, \% & 25 & 38 & $<0.001$ \\
\hline ATS pack-years & $47.4(26.7)$ & $49.9(23.6)$ & 0.04 \\
\hline $\mathrm{FEV}_{1}$ & $2.0(1.0)$ & $3.4(1.0)$ & $<0.001$ \\
\hline $\mathrm{FEV}_{1} \%$ predicted & $68(30)$ & $73(26)$ & $<0.001$ \\
\hline $\mathrm{FEV}_{1} / \mathrm{FVC}$ & $58(20)$ & $62(16)$ & $<0.001$ \\
\hline Change in $\mathrm{FEV}_{1}$ with bronchodilators, \% & $7.3(10)$ & $13.6(13)$ & $<0.001$ \\
\hline Emphysema, \%* & $9.5(11.7)$ & $8.1(10.1)$ & 0.01 \\
\hline SGRQ & $28.2(23.2)$ & $32.0(19.9)$ & $<0.001$ \\
\hline BODE & $1.8(2.1)$ & $1.4(1.9)$ & $<0.001$ \\
\hline $\mathrm{Sp}_{\mathrm{O}_{2}}$, resting & $93.9(3.7)$ & $95.5(2.8)$ & $<0.001$ \\
\hline Chronic bronchitis, \% & 19 & 20 & 0.43 \\
\hline Duration of follow-up, yr & $4.0(1.1)$ & $2.3(0.8)$ & \\
\hline PRISm & 1.5 & N/A & \\
\hline Healthy smokers & 39.5 & 31.6 & \\
\hline \multicolumn{4}{|l|}{ GOLD stage, \% } \\
\hline Stage 1 & 1.2 & 16.9 & \\
\hline Stage 2 & 24.2 & 29.5 & \\
\hline Stage 3 & 21.5 & 16.1 & \\
\hline Stage 4 & 12.1 & 5.9 & \\
\hline Subjects with exacerbation in prior year, \% & 30 & 24 & $<0.001$ \\
\hline Subjects with severe exacerbation in prior year, \% & 11 & 11 & 0.47 \\
\hline
\end{tabular}

Definition of abbreviations: ATS = American Thoracic Society; BMI = body mass index; BODE = BMI, airflow obstruction, dyspnea, and exercise index (42); COPDGene = Genetic Epidemiology of COPD; GOLD = Global Initiative for Chronic Obstructive Lung Disease; N/A = not applicable; PRISm = preserved ratio, impaired spirometry; SGRQ = St. George's Respiratory Questionnaire; SPIROMICS = Subpopulations and Intermediate Outcomes Measures in COPD Study; $\mathrm{Sp}_{\mathrm{o}_{2}}=$ oxygen saturation as measured by pulse.

PRISm: $\mathrm{FEV}_{1} / \mathrm{FVC}^{2}>0.7 ; \mathrm{FEV}_{1}<80 \%$.

Data are shown as mean (SD) unless otherwise indicated.

*\% Emphysema defined as $-950 \mathrm{HU}$.

$P<0.001)$. These differences are reflected in the higher percentage of subjects in SPIROMICS with mild COPD (GOLD stage 1) compared with COPDGene (16.9 vs. $1.2 \%$, respectively).

SPIROMICS subjects were followed for $2.28 \pm 0.8$ years (mean $\pm \mathrm{SD}$ ), whereas the COPDGene cohort was followed for $4.04 \pm$ 1.1 years. At baseline, the total retrospective exacerbation rate was slightly lower in SPIROMICS subjects, likely reflecting a greater fraction of subjects with less severe disease, but the rate of severe retrospective exacerbations was similar between cohorts (Table 2). Overall, SPIROMICS had a lower prospective exacerbation rate, but this finding varied across GOLD stages. Rates of severe prospective exacerbations were also lower in SPIROMICS.

\section{Biomarker Associations}

We performed separate extensive analyses for both cohorts, and a metaanalysis (Supplemental File E1). These biomarker analyses were conducted on total and severe for both retrospective and prospective exacerbations, with and without clinical covariates on all subjects (Supplemental File E1, Tabs E1-E8). The analysis was repeated on a subset consisting of GOLD stage 2-4 subjects (Supplemental File E1, Tabs E9-E12). In most analyses, a small percentage of biomarkers were significant in each cohort. However, rarely were biomarkers significant in the metaanalyses. It became obvious that, regardless the type of exacerbation or population, results in one cohort were not reflected well in the other cohort. In most cases, inclusion of clinical covariates in the models further reduced the number of significant findings (e.g., for all prospective exacerbations with vs. without covariates) (Figure 1). As expected, clinical features were individually significant in both cohorts for predicting exacerbations as exemplified by the analysis for total prospective exacerbations (Table E2).

\section{Blood Biomarkers Associated with Total and Severe Retrospective Exacerbation}

Without including any clinical covariates, only one biomarker was significantly associated with a history of severe retrospective exacerbation. $\alpha_{1}$-Antitrypsin (A1AT; SERPINA1) was statistically significant in both the COPDGene cohort and in the metaanalysis, but not in the SPIROMICS cohort (Supplemental File E1, Tabs E9 and E10). When including clinical covariates, no biomarkers were significantly associated with total retrospective exacerbations in the SPIROMICS cohort or the metaanalysis, but two biomarkers were significant in COPDGene (Table E3 and Supplemental File E1, Tab 1). When considering only severe retrospective exacerbations, we found four statistically significant biomarkers in SPIROMICS and two in COPDGene (Table E4). Only one of these biomarkers was significant in both cohorts (ICAM1), but was not significant in the metaanalysis (Supplemental File E1, Tab E2).

In subjects with more severe COPD (GOLD stages 2-4), no biomarkers in either cohort were significantly associated with total retrospective exacerbations (Table E5). In this subgroup, we did identify significant associations with severe exacerbations in the COPDGene cohort for five biomarkers 
Table 2. Exacerbations

\begin{tabular}{|c|c|c|c|c|c|c|}
\hline & \multicolumn{3}{|c|}{ Retrospective } & \multicolumn{3}{|c|}{ Prospective } \\
\hline & COPDGene & SPIROMICS & $P$ Value & COPDGene & SPIROMICS & $P$ Value \\
\hline Exacerbations, $\mathrm{n}$ & 359 & 614 & & 1,650 & 1,821 & \\
\hline Exacerbation rate ${ }^{*}$ & 0.601 & 0.401 & 0.002 & 0.866 & 0.601 & $<0.001$ \\
\hline GOLD stage $1^{*}$ & 0.857 & 0.190 & 0.466 & 0.156 & 0.277 & 0.003 \\
\hline GOLD stage $2^{*}$ & 0.667 & 0.400 & 0.013 & 1.286 & 0.752 & 0.003 \\
\hline GOLD stage $3^{*}$ & 0.908 & 0.718 & 0.130 & 2.029 & 1.425 & 0.049 \\
\hline GOLD stage $4^{*}$ & 1.423 & 1.011 & 0.102 & 1.530 & 2.806 & 0.744 \\
\hline Healthy smoker exacerbation rate ${ }^{*}$ & 0.121 & 0.241 & 0.008 & 0.233 & 0.212 & 0.321 \\
\hline Severe exacerbations, $\mathrm{n}$ & 98 & 238 & & 479 & 565 & \\
\hline Severe exacerbation rate $^{*}$ & 0.164 & 0.155 & 0.746 & 0.251 & 0.189 & 0.005 \\
\hline GOLD stage $1^{*}$ & 0.0 & 0.085 & 0.015 & 0.125 & 0.082 & 0.545 \\
\hline GOLD stage $2^{*}$ & 0.135 & 0.135 & 0.999 & 0.341 & 0.192 & 0.033 \\
\hline GOLD stage $3^{*}$ & 0.223 & 0.270 & 0.487 & 0.579 & 0.514 & 0.356 \\
\hline GOLD stage $4^{*}$ & 0.535 & 0.571 & 0.820 & 0.670 & 0.998 & 0.930 \\
\hline Healthy smoker severe exacerbation rate* & 0.029 & 0.077 & 0.052 & 0.041 & 0.057 & 0.985 \\
\hline
\end{tabular}

Definition of abbreviations: COPDGene = Genetic Epidemiology of COPD; GOLD = Global Initiative for Chronic Obstructive Lung Disease; SPIROMICS = Subpopulations and Intermediate Outcomes Measures in COPD Study.

*Exacerbation rate: calculated as number of exacerbations divided by number of patients and then adjusted for years of follow-up.

(sRAGE, brain-derived neurotrophic factor, plasminogen activator inhibitor-1, CXCL5, and A1AT) (Table E6), but none were significant within the SPIROMICS cohort. In the metaanalysis, six biomarkers were significantly associated with severe retrospective exacerbations (Supplemental File E1, Tab E6).

\section{Biomarkers Predictive of Future Exacerbations}

The two cohorts yielded a robust amount of follow-up data to permit association of baseline biomarker values to future exacerbations: 3,653 person-years in SPIROMICS and 2,739 person-years in COPDGene. Moreover, a substantial fraction of our subjects had exacerbations. Collectively, $58.2 \%$ of subjects with COPD and $19.4 \%$ of smokers without airflow obstruction reported at least one respiratory event requiring antibiotics and/or prednisone during the follow-up period, and $29.9 \%$ of subjects with COPD and $6.8 \%$ of smokers without airflow obstruction reported a severe exacerbation.

Without clinical covariates in the model, the COPDGene cohort identified multiple associations of baseline biomarkers with future exacerbations (19 with total, 13 with severe) (Supplemental File E1, Tabs E11 and E12), whereas the SPIROMICS cohort found two biomarkers each associated with total exacerbations and severe exacerbations (Supplemental File E1, Tabs E11 and E12). Once again, however, when including clinical variables in the prediction model, no baseline biomarkers significantly associated with future total or severe exacerbations in prospective followup (Table E7 and Supplemental File E1, Tab E3). In the metaanalysis, two biomarkers were associated with prospective severe exacerbations; one in COPDGene, and none in the SPIROMICS cohort (Table 3 and Supplemental File E1, Tab E4). Of these, only decorin (DCN) was significant in both COPDGene and the metaanalysis $(\mathrm{FDR}=$ 0.001; $P<0.001) . \alpha_{2}$-Macroglobulin $(\mathrm{A} 2 \mathrm{M})(\mathrm{FDR}=0.011 ; P<0.001)$ was significant only in the metaanalysis.

In subjects with more severe COPD (GOLD stages 2-4), none of the biomarkers were significantly associated with future total exacerbations (Table E8). When looking only at severe exacerbations, DCN (FDR $=0.015 ; P<0.001)$ remained positively associated with severe exacerbations both in the COPDGene cohort and in the metaanalysis (Table 4). By contrast, the SPIROMICS cohort identified associations of future severe exacerbations with five biomarkers: A1AT and ferritin (both FDR $=0.010 ; P<0.001$ ), CXCL10 and CXCL9 (both FDR $=0.028$; $P<0.001)$, and CCL11 $(\mathrm{FDR}=0.028$; $P<0.002$ ).

A Cox proportional hazards model was used to assess associations between biomarkers and time-to-next exacerbation. In this model, none of the adjusted hazards ratios differed significantly from 1 in both cohorts for total exacerbations. sRAGE trended toward a significant association in SPIROMICS for severe exacerbations, but these results were not replicated in COPDGene (APPENDIX in the online supplement, Figure E2, and Tables E9 and E10).

To estimate the effect size for each biomarker, we calculated a pseudo $R^{2}$ value to determine whether each biomarker improved the ability to detect the outcome when compared with clinical covariates. Clinical predictors accounted for the majority of variability seen in our model, but some of the biomarkers did marginally improve (additional 1-2\%) the model's ability to predict the next exacerbation above clinical covariates alone (Tables E11-E14).

To evaluate the possibility that a panel of biomarkers may be more predictive than individual biomarkers across the two studies, stepwise multinomial regression was performed for each cohort using significant univariate factors for total severe prospective exacerbations. These analyses demonstrated that clinical predictors were relatively preserved between cohorts, whereas with the exception of A1AT, biomarkers did not replicate (Tables E15 and E16).

\section{Discussion}

This analysis of two large cohorts of extensively phenotyped ever-smokers 

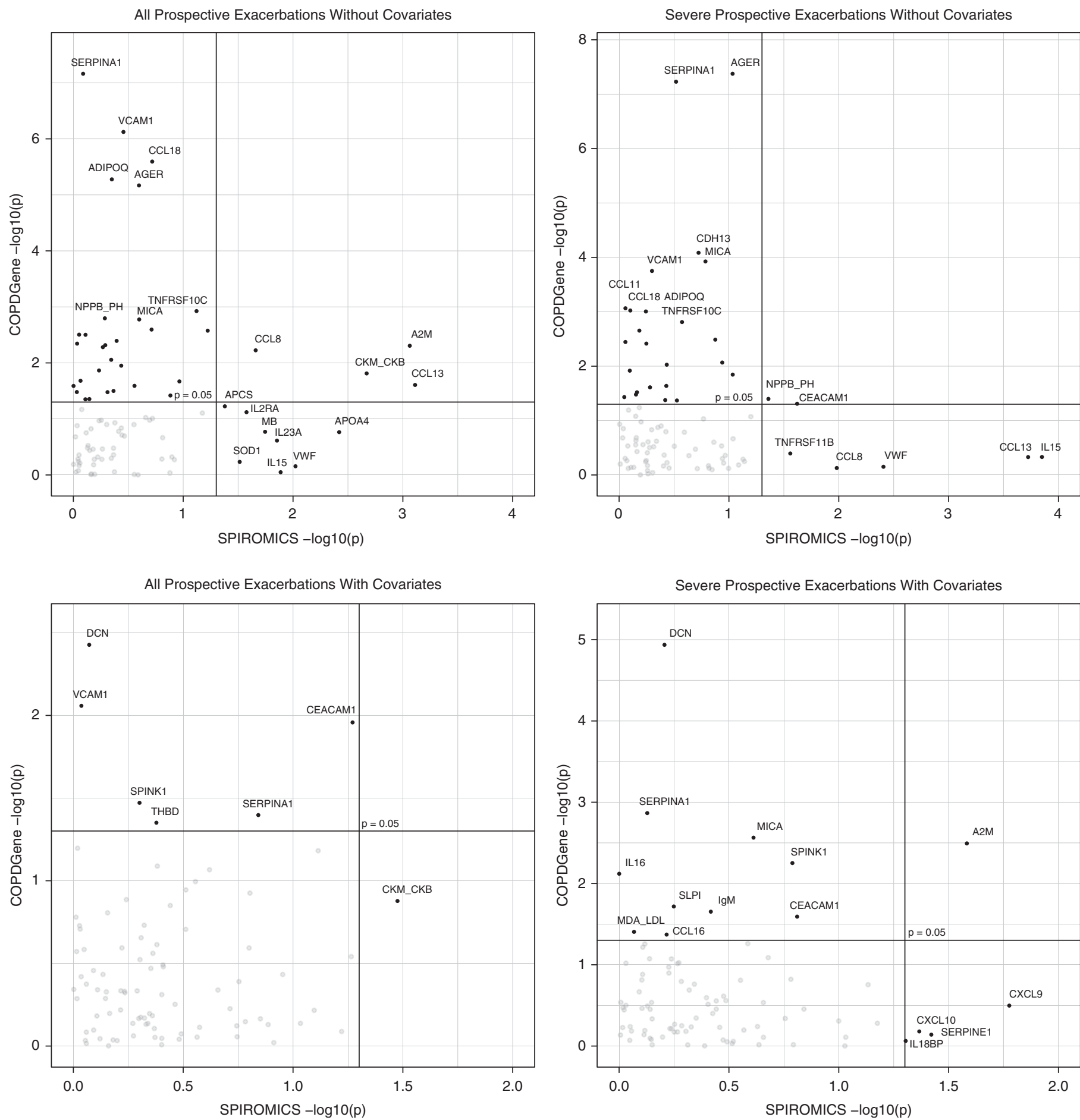

Figure 1. Scatter plot of $P$ values for biomarkers. $P$ values of each biomarker were plotted for the negative binomial regression using the model developed with covariates as described in the MetHods section and without covariates. The dark lines represent $P=0.05$. COPDGene $=$ Genetic Epidemiology of COPD; SPIROMICS = Subpopulations and Intermediate Outcomes Measures in COPD Study.

without and with airflow obstruction (SPIROMICS and COPDGene) used nearly identical methodology to measure 90 plasma biomarkers and to determine their associations with previous and future respiratory events reported during careful longitudinal follow-up. By comparing results within the individual cohorts and in a metaanalysis, we demonstrate two key finding regarding blood biomarkers obtained in the stable state: first, despite statistically significant associations within a given cohort or in metaanalysis of some biomarkers with exacerbations (independent of established clinical 
Table 3. Negative Binomial Regression Severe Exacerbations (Prospective)

\begin{tabular}{lrrrrrrrr}
\hline & \multicolumn{3}{c}{ SPIROMICS } & & \multicolumn{3}{c}{ COPDGene } \\
\cline { 2 - 3 } \cline { 6 - 7 } Biomarker & $\boldsymbol{\beta}$ Coefficient & $\boldsymbol{P}$ Value & FDR & & $\boldsymbol{\beta}$ Coefficient & $\boldsymbol{P}$ Value & FDR \\
A2M & 0.247 & 0.027 & 0.893 & & 0.461 & 0.003 & 0.072 \\
CCL16 & -0.047 & 0.599 & 0.914 & & 0.317 & 0.043 & 0.348 \\
CEACAM1 & 0.113 & 0.152 & 0.914 & & 0.385 & 0.026 & 0.256 \\
CXCL10 & 0.194 & 0.044 & 0.893 & & 0.080 & 0.661 & 0.865 \\
CXCL9 & 0.252 & 0.017 & 0.893 & & 0.165 & 0.318 & 0.673 \\
DCN & 0.047 & 0.622 & 0.929 & & 0.585 & 0.000 & 0.001 \\
IgM & -0.079 & 0.384 & 0.914 & & 0.307 & 0.022 & 0.250 \\
IL16 & 0.000 & 0.998 & 0.998 & & -0.383 & 0.008 & 0.114 \\
IL18BP & 0.168 & 0.050 & 0.893 & & 0.023 & 0.865 & 0.961 \\
MDA_LDL_log & 0.057 & 0.865 & 0.985 & & 0.838 & 0.039 & 0.348 \\
MICA_log & -0.259 & 0.250 & 0.914 & & 0.838 & 0.003 & 0.072 \\
SERPINA1 & -0.030 & 0.743 & 0.929 & & 0.521 & 0.001 & 0.061 \\
SERPINE1 & -0.196 & 0.038 & 0.893 & & 0.062 & 0.724 & 0.903 \\
SLPI & -0.055 & 0.561 & 0.914 & & -0.317 & 0.019 & 0.246 \\
SPINK1 & 0.118 & 0.160 & 0.914 & & -0.333 & 0.006 & 0.101 \\
\hline
\end{tabular}

Definition of abbreviations: COPDGene = Genetic Epidemiology of COPD; FDR = false discovery rate; SPIROMICS = Subpopulations and Intermediate Outcomes Measures in COPD Study.

*Log denotes that variables were transformed to binary yes or no based on serum levels above or below lower limit of quantitation of assay.

predictors, including history of previous exacerbation), that those associations replicated poorly between cohorts; and second, that biomarkers only marginally improved the predictive value of easily obtained clinical variables. Given the magnitudes of follow-up ( $>6,300$ personyears), and of events, which among subjects with COPD occurred in almost $60 \%$ (any exacerbation) and 30\% (severe exacerbation), it is unlikely that these findings reflect a lack of statistical power. Instead, our results imply the need for very considerable additional investigation of the basic biology of exacerbations, which these two studies recently showed afflict not only those who meet current definitions of COPD, but are also a feature of some smokers without airflow obstruction $(29,30)$.

These results provide a novel insight into the shortcomings of the method by which many previous studies attempted to identify biomarkers predictive of exacerbations. Although almost all published work reported some positive associations of biomarker with exacerbations, some of these studies reported biomarkers associated with exacerbations $(31,32)$ or during recovery (33), but few included validation in independent cohorts and prospective analysis of risk of future exacerbations, or discussion of the effect size of individual biomarkers. Each of these features is addressed in the current study. Examples of biomarkers associated with exacerbation risk in previous studies include CRP, IL-6, fibrinogen, white blood cell count, surfactant protein $\mathrm{D}$, chemokine (C-C motif) ligand 18 (PARC), CC16, and blood eosinophils $(34,35)$. Most of these biomarkers were identified in the ECLIPSE study, without large-scale replication $(1,5,6)$.

In agreement with previous studies, we also identified several biomarkers associated with a history of previous exacerbations. Thus, inflammatory signatures likely remain an important feature of subjects with COPD who report exacerbations. However, we extend the field by demonstrating how few biomarkers, independent of clinical variables, were predictive of the outcome of future exacerbations. Moreover, those biomarkers that we did identify in SPIROMICS and COPDGene (e.g., DCN, A2M, A1AT, sRAGE) differed from results of ECLIPSE, even after creating subgroup analyses to specifically replicate the ECLIPSE cohort. Importantly, after adjustment for key clinical covariates, neither COPDGene nor SPIROMICS identified fibrinogen as a predictive biomarker of exacerbations. Nevertheless, we confirmed the predictive value of clinical variables (history of prior exacerbation, dyspnea score, spirometry, and gastroesophageal reflux disease), which replicated significantly between our cohorts to predict future exacerbations.
There are several possible reasons for such discrepant results in the biomarker field. One key candidate is the heterogeneous nature of exacerbations, which can be triggered by myriad factors. Approximately half of exacerbations have been associated with bacterial infections by culture-dependent techniques and nearly $60 \%$ with viral infections (36), but some exacerbations may be triggered by air pollution (37), changes in temperature and barometric pressure $(38,39)$, and possibly shifts in intrapulmonary fluids in those with occult heart failure (40). From this viewpoint, the inability to identify reproducible biomarkers follows inability to classify the etiology for each exacerbation accurately. Thus, with advances in molecular diagnostics (e.g., NextGen Sequencing, microbiome analysis) it may be possible to subtype exacerbation triggers more accurately, and accordingly, to find biomarkers indicating susceptibility to individual triggers. However, it is also possible that no single or even combination of plasma proteins measured in the stable state will predict these highly stochastic events. Thus, it may be necessary to develop practical means to assess the function of cell types within the lungs themselves, especially airway epithelium and immune cell types. An alternative would be to examine the response of plasma biomarkers to an evoked phenotype, or of blood cells to stimulation ex vivo.

We considered potential factors that might contribute to lack of reproducibility between our cohorts, including differences in clinical variables, such as current smoking status, proportion of patients with concurrent asthma, or geography. However, the SPIROMICS and COPDGene cohorts have many common features, including their design, congruent follow-up methods, and some (but not all) shared clinical sites at which the same investigators recruit to both studies. These two cohorts are generally more similar to each other than to other cohorts, including ECLIPSE, designed to address different questions. Important features of the current analysis are nearly identical biomarker platform assessments, adjustment for known clinical covariates, and sufficient power (large number of exacerbations). We are confident that the populations and biomarker platforms were comparable because a recent publication showed nearly identical identification of genetic markers for biomarkers (18). 
Table 4. Negative Binomial Regression GOLD Stage 2-4 Severe Exacerbations (Prospective)

\begin{tabular}{|c|c|c|c|c|c|c|}
\hline \multirow[b]{2}{*}{ Biomarker } & \multicolumn{3}{|c|}{ SPIROMICS } & \multicolumn{3}{|c|}{ COPDGene } \\
\hline & 及 Coefficient & $P$ Value & FDR & $\beta$ Coefficient & $P$ Value & FDR \\
\hline A2M & 0.179 & 0.276 & 0.672 & 0.484 & 0.006 & 0.169 \\
\hline ANGPT1 & 0.173 & 0.201 & 0.603 & -0.293 & 0.048 & 0.242 \\
\hline BDNF & 0.222 & 0.122 & 0.508 & -0.373 & 0.025 & 0.242 \\
\hline CA9_log* & 0.213 & 0.502 & 0.822 & 0.637 & 0.029 & 0.242 \\
\hline CCL11_log* & 0.770 & 0.002 & 0.028 & 0.523 & 0.104 & 0.360 \\
\hline $\mathrm{CCL}_{13}$ & 0.382 & 0.008 & 0.109 & -0.241 & 0.141 & 0.387 \\
\hline CCL18 & 0.095 & 0.511 & 0.822 & 0.351 & 0.041 & 0.242 \\
\hline CCL20_log* & 0.611 & 0.017 & 0.160 & 0.259 & 0.487 & 0.742 \\
\hline CCL4 & 0.140 & 0.315 & 0.727 & -0.580 & 0.003 & 0.152 \\
\hline CCL5 & 0.259 & 0.127 & 0.508 & -0.379 & 0.026 & 0.242 \\
\hline CCL8 & 0.271 & 0.018 & 0.160 & -0.037 & 0.798 & 0.923 \\
\hline $\mathrm{CDH} 1$ & 0.050 & 0.690 & 0.900 & 0.318 & 0.044 & 0.242 \\
\hline $\mathrm{CDH} 13$ & 0.315 & 0.016 & 0.160 & 0.065 & 0.700 & 0.900 \\
\hline CEACAM1 & 0.246 & 0.034 & 0.252 & 0.241 & 0.128 & 0.387 \\
\hline CXCL10 & 0.407 & 0.001 & 0.028 & 0.103 & 0.552 & 0.790 \\
\hline CXCL9 & 0.463 & 0.001 & 0.028 & 0.175 & 0.329 & 0.590 \\
\hline DCN & 0.101 & 0.379 & 0.796 & 0.592 & 0.000 & 0.006 \\
\hline FTL_FTH1 & -0.479 & 0.000 & 0.010 & -0.053 & 0.767 & 0.920 \\
\hline $\lg M$ & -0.089 & 0.471 & 0.822 & 0.327 & 0.033 & 0.242 \\
\hline IL12B_log* & 0.496 & 0.089 & 0.471 & 0.663 & 0.039 & 0.242 \\
\hline IL16 & 0.104 & 0.420 & 0.796 & -0.364 & 0.027 & 0.242 \\
\hline IL18BP & 0.312 & 0.027 & 0.220 & 0.086 & 0.562 & 0.790 \\
\hline IL8 & 0.350 & 0.006 & 0.087 & 0.256 & 0.166 & 0.427 \\
\hline LTF & -0.229 & 0.085 & 0.471 & -0.294 & 0.054 & 0.245 \\
\hline MDA_LDL_log* & 0.480 & 0.274 & 0.672 & 1.031 & 0.014 & 0.242 \\
\hline MICA_log ${ }^{*}$ & 0.284 & 0.369 & 0.796 & 0.675 & 0.033 & 0.242 \\
\hline NPPB_PH & -0.048 & 0.741 & 0.909 & 0.309 & 0.048 & 0.242 \\
\hline SERPINA1 & -0.373 & 0.000 & 0.010 & 0.367 & 0.031 & 0.242 \\
\hline SLPI & -0.203 & 0.178 & 0.562 & -0.264 & 0.055 & 0.245 \\
\hline
\end{tabular}

Definition of abbreviations: COPDGene = Genetic Epidemiology of COPD; FDR = false discovery rate; GOLD = Global Initiative for Chronic Obstructive Lung Disease; SPIROMICS = Subpopulations and Intermediate Outcomes Measures in COPD Study.

*Log denotes that variables were transformed to binary yes or no based on serum levels above or below lower limit of quantitation of assay.

Despite these strengths, there remain limitations. Exacerbations were reported at 3- or 6-month intervals and relied on self-reporting. This method could lead to underreporting of mild or moderate events, but is unlikely to influence identification of severe exacerbations (41). Because of our follow-up design, we could not study the seasonal nature of exacerbations. There are statistical limitations (or strengths), such as the requirement to adjust for multiple comparisons using this large biomarker panel. Additionally some biomarkers, particularly low abundance interleukins, such as IL-6, were below the limit of detection in the RBM assays and required analysis on other assay platforms. Most importantly, neither cohort has detailed information, such as viral or bacterial respiratory cultures, or biomarker collection during events.

In summary, this analysis identified several biomarkers associated with exacerbation history and exacerbation risk, but there was poor reproducibility of specific biomarkers between cohorts. Ultimately, we show that clinical factors remain the strongest current predictors of future exacerbations. Improved clinical utility of blood biomarkers to predict COPD exacerbations awaits methodology to reduce heterogeneity of events, possibly by categorizing their triggers, and this may not be attainable by testing in the stable state.

Author disclosures are available with the tex of this article at www.atsjournals.org.

\begin{abstract}
Acknowledgment: The authors thank the SPIROMICS (Subpopulations and Intermediate Outcomes Measures in COPD Study) participants and participating physicians, investigators, and staff for making this research possible. More information about the study and how to access SPIROMICS data is at www.spiromics.org. The project officers from the Lung Division of the NHLBI were Lisa Postow, Ph.D., and Thomas Croxton, Ph.D., M.D.
\end{abstract}

\section{Current and former investigators of the} SPIROMICS sites and reading centers: Neil E. Alexis, Ph.D.; Wayne H. Anderson, Ph.D.; R. Graham Barr, M.D., Dr.P.H.; Eugene R. Bleecker, M.D.; Richard C. Boucher, M.D.; Russell P. Bowler, M.D., Ph.D.; Elizabeth E. Carretta, M.P.H.; Stephanie A. Christenson, M.D.; Alejandro P. Comellas, M.D.;

Christopher B. Cooper, M.D., Ph.D.

David J. Couper, Ph.D.; Gerard J. Criner, M.D.; Ronald G. Crystal, M.D.; Jeffrey L. Curtis, M.D.; Claire M. Doerschuk, M.D.; Mark T. Dransfield, M.D.; Christine M. Freeman, Ph.D.; MeiLan K. Han, M.D., M.S.; Nadia N. Hansel, M.D., M.P.H.; Annette T. Hastie, Ph.D.; Eric A. Hoffman, Ph.D.; Robert J. Kaner, M.D.; Richard E. Kanner, M.D.; Eric C. Kleerup, M.D.; Jerry A. Krishnan, M.D., Ph.D.

Lisa M. LaVange, Ph.D.; Stephen C. Lazarus, M.D.; Fernando J. Martinez, M.D., M.S.; Deborah A. Meyers, Ph.D.; John D. Newell, Jr., M.D.; Elizabeth C. Oelsner, M.D.

M.P.H.; Wanda K. O'Neal, Ph.D.; Robert Paine III, M.D.; Nirupama Putcha, M.D., M.H.S.; Stephen I. Rennard, M.D.; Donald P. Tashkin, M.D.; Mary Beth Scholand, M.D.; J. Michael Wells, M.D.; Robert A. Wise, M.D.; and Prescott G. Woodruff, M.D., M.P.H.

\section{References}

1. Hurst JR, Vestbo J, Anzueto A, Locantore N, Müllerova $\mathrm{H}$, Tal-Singer R, Miller B, Lomas DA, Agusti A, Macnee W, et al.; Evaluation of COPD Longitudinally to Identify Predictive Surrogate Endpoints (ECLIPSE) Investigators. Susceptibility to exacerbation in chronic obstructive pulmonary disease. N Engl J Med 2010;363: 1128-1138.
2. Agustí A, Faner R. Systemic inflammation and comorbidities in chronic obstructive pulmonary disease. Proc Am Thorac Soc 2012; 9:43-46.

3. Bowler RP, Kim V, Regan E, Williams AA, Santorico SA, Make BJ, Lynch DA, Hokanson JE, Washko GR, Bercz P, et al.; COPDGene investigators. Prediction of acute respiratory disease in current and former smokers with and without COPD. Chest 2014;146: 941-950. 
4. Donaldson GC, Wedzicha JA. COPD exacerbations. 1: Epidemiology. Thorax 2006;61:164-168.

5. Duvoix A, Dickens J, Haq I, Mannino D, Miller B, Tal-Singer R, Lomas DA. Blood fibrinogen as a biomarker of chronic obstructive pulmonary disease. Thorax 2013;68:670-676.

6. Thomsen M, Ingebrigtsen TS, Marott JL, Dahl M, Lange P, Vestbo J, Nordestgaard BG. Inflammatory biomarkers and exacerbations in chronic obstructive pulmonary disease. JAMA 2013;309:2353-2361.

7. Dickens JA, Miller BE, Edwards LD, Silverman EK, Lomas DA, Tal-Singer R; Evaluation of COPD Longitudinally to Identify Surrogate Endpoints (ECLIPSE) study investigators. COPD association and repeatability of blood biomarkers in the ECLIPSE cohort. Respir Res 2011;12:146.

8. Ozyurek BA, Ulasli SS, Bozbas SS, Bayraktar N, Akcay S. Value of serum and induced sputum surfactant protein-D in chronic obstructive pulmonary disease. Multidiscip Respir Med 2013;8:36.

9. Minas M, Mystridou P, Georgoulias P, Pournaras S, Kostikas K, Gourgoulianis KI. Fetuin-A is associated with disease severity and exacerbation frequency in patients with COPD. COPD 2013;10:28-34.

10. Kirdar S, Serter M, Ceylan E, Sener AG, Kavak T, Karadağ F. Adiponectin as a biomarker of systemic inflammatory response in smoker patients with stable and exacerbation phases of chronic obstructive pulmonary disease. Scand J Clin Lab Invest 2009;69:219-224.

11. Miller BE, Tal-Singer R, Rennard SI, Furtwaengler A, Leidy N, Lowings M, Martin UJ, Martin TR, Merrill DD, Snyder J, et al.; Perspective of the Chronic Obstructive Pulmonary Disease Biomarker Qualification Consortium. Plasma fibrinogen qualification as a drug development tool in chronic obstructive pulmonary disease. Am J Respir Crit Care Med 2016;193:607-613.

12. Couper D, LaVange LM, Han M, Barr RG, Bleecker E, Hoffman EA, Kanner R, Kleerup E, Martinez FJ, Woodruff PG, et al.; SPIROMICS Research Group. Design of the Subpopulations and Intermediate Outcomes in COPD Study (SPIROMICS). Thorax 2014;69:491-494.

13. Celli BR, MacNee W, ATS/ERS Task Force. Standards for the diagnosis and treatment of patients with COPD: a summary of the ATS/ERS position paper. Eur Respir J 2004;23:932-946.

14. Regan EA, Hokanson JE, Murphy JR, Make B, Lynch DA, Beaty TH, Curran-Everett D, Silverman EK, Crapo JD. Genetic epidemiology of COPD (COPDGene) study design. COPD 2010;7:32-43.

15. Carolan BJ, Kim YI, Williams AA, Kechris K, Lutz S, Reisdorph N, Bowler RP. The association of adiponectin with computed tomography phenotypes in chronic obstructive pulmonary disease. Am J Respir Crit Care Med 2013;188:561-566.

16. O'Neal WK, Anderson W, Basta PV, Carretta EE, Doerschuk CM, Barr RG, Bleecker ER, Christenson SA, Curtis JL, Han MK, et al.; SPIROMICS Investigators. Comparison of serum, EDTA plasma and P100 plasma for Luminex-based biomarker multiplex assays in patients with chronic obstructive pulmonary disease in the SPIROMICS study. J Transl Med 2014;12:9.

17. Carolan BJ, Hughes G, Morrow J, Hersh CP, O'Neal WK, Rennard S, Pillai SG, Belloni P, Cockayne DA, Comellas AP, et al. The association of plasma biomarkers with computed tomographyassessed emphysema phenotypes. Respir Res 2014;15:127.

18. Sun W, Kechris K, Jacobson S, Drummond MB, Hawkins GA, Yang J, Chen TH, Quibrera PM, Anderson W, Barr RG, et al.; SPIROMICS Research Group; COPDGene Investigators. Common genetic polymorphisms influence blood biomarker measurements in COPD. PLOS Genet 2016;12:e1006011.

19. Fabbri LM, Hurd SS, GOLD Scientific Committee. Global strategy for the diagnosis, management and prevention of COPD: 2003 update. Eur Respir J 2003;22:1-2.

20. Wan ES, Castaldi PJ, Cho MH, Hokanson JE, Regan EA, Make BJ, Beaty TH, Han MK, Curtis JL, Curran-Everett D, et al.; COPDGene Investigators. Epidemiology, genetics, and subtyping of preserved ratio impaired spirometry (PRISm) in COPDGene. Respir Res 2014;15:89.

21. Jones PW, Quirk FH, Baveystock CM, Littlejohns P. A self-complete measure of health status for chronic airflow limitation. The St. George's Respiratory Questionnaire. Am Rev Respir Dis 1992;145:1321-1327.

22. Kim V, Han MK, Vance GB, Make BJ, Newell JD, Hokanson JE, Hersh CP, Stinson D, Silverman EK, Criner GJ; COPDGene Investigators. The chronic bronchitic phenotype of COPD: an analysis of the COPDGene Study. Chest 2011;140:626-633.
23. Team RCR. A language and environment for statistical computing. 3.2.4 ed. Vienna, Austria: R Foundation for Statistical Computing; 2015.

24. Cameron AC, Trivedi PK. Regression analysis of count data. Cambridge, UK: Cambridge University Press; 1998.

25. Zeileis A, Kleiber $C$, Jackman $S$. Regression models for count data in $R$. $J$ Stat Softw 2008;27:1-25.

26. Andersen PK, Gill RD. Cox's regression model for counting processes: a large sample study. Ann Statist 1982;10:1100-1120.

27. Therneau TM, Grambsch PM. Modeling survival data: extending the Cox model. New York: Springer; 2000.

28. Cragg JG, Uhler RS. The demand for automobiles. Can J Econ 1970;3: 386-406.

29. Regan EA, Lynch DA, Curran-Everett D, Curtis JL, Austin JH, Grenier PA, Kauczor HU, Bailey WC, DeMeo DL, Casaburi RH, et al.; Genetic Epidemiology of COPD Investigators. Clinical and radiologic disease in smokers with normal spirometry. JAMA Intern Med 2015;175: 1539-1549.

30. Woodruff PG, Barr RG, Bleecker E, Christenson SA, Couper D, Curtis JL, Gouskova NA, Hansel NN, Hoffman EA, Kanner RE, et al.; SPIROMICS Research Group. Clinical significance of symptoms in smokers with preserved pulmonary function. N Engl J Med 2016; 374:1811-1821.

31. Hurst JR, Donaldson GC, Perera WR, Wilkinson TM, Bilello JA, Hagan GW, Vessey RS, Wedzicha JA. Use of plasma biomarkers at exacerbation of chronic obstructive pulmonary disease. Am J Respir Crit Care Med 2006;174:867-874.

32. Karadag F, Karul AB, Cildag O, Yilmaz M, Ozcan H. Biomarkers of systemic inflammation in stable and exacerbation phases of COPD. Lung 2008;186:403-409.

33. Perera WR, Hurst JR, Wilkinson TM, Sapsford RJ, Müllerova $H$, Donaldson GC, Wedzicha JA. Inflammatory changes, recovery and recurrence at COPD exacerbation. Eur Respir J 2007;29:527-534.

34. Pascoe S, Locantore N, Dransfield MT, Barnes NC, Pavord ID. Blood eosinophil counts, exacerbations, and response to the addition of inhaled fluticasone furoate to vilanterol in patients with chronic obstructive pulmonary disease: a secondary analysis of data from two parallel randomised controlled trials. Lancet Respir Med 2015;3: 435-442.

35. Vedel-Krogh S, Nielsen SF, Lange P, Vestbo J, Nordestgaard BG. Blood eosinophils and exacerbations in chronic obstructive pulmonary disease. The Copenhagen General Population Study. Am J Respir Crit Care Med 2016;193:965-974.

36. Sethi S, Murphy TF. Infection in the pathogenesis and course of chronic obstructive pulmonary disease. N Engl J Med 2008;359:2355-2365.

37. Song Q, Christiani DC, XiaorongWang, Ren J. The global contribution of outdoor air pollution to the incidence, prevalence, mortality and hospital admission for chronic obstructive pulmonary disease: a systematic review and meta-analysis. Int J Environ Res Public Health 2014:11:11822-11832.

38. Almagro P, Hernandez C, Martinez-Cambor P, Tresserras R, Escarrabill J. Seasonality, ambient temperatures and hospitalizations for acute exacerbation of COPD: a population-based study in a metropolitan area. Int J Chron Obstruct Pulmon Dis 2015;10:899-908.

39. Tseng CM, Chen YT, Ou SM, Hsiao YH, Li SY, Wang SJ, Yang AC, Chen TJ, Perng DW. The effect of cold temperature on increased exacerbation of chronic obstructive pulmonary disease: a nationwide study. PLoS One 2013;8:e57066.

40. Marcun R, Stankovic I, Vidakovic R, Farkas J, Kadivec S, Putnikovic B, Ilic I, Neskovic AN, Lainscak M. Prognostic implications of heart failure with preserved ejection fraction in patients with an exacerbation of chronic obstructive pulmonary disease. Intern Emerg Med 2016;11:519-527.

41. Frei A, Siebeling L, Wolters C, Held L, Muggensturm P, Strassmann A, Zoller M, Riet GT, Puhan MA. The inaccuracy of patient recall for COPD exacerbation rate estimation and its implications: results from central adjudication. Chest 2016;150:860-868.

42. Celli BR, Cote CG, Marin JM, Casanova C, Montes de Oca M, Mendez RA, Pinto Plata V, Cabral HJ. The body-mass index, airflow obstruction, dyspnea, and exercise capacity index in chronic obstructive pulmonary disease. N Engl J Med 2004;350:1005-1012. 